\title{
25 Years of Quantum Groups: from Definition to Classification
}

A. Stolin

In mathematics and theoretical physics, quantum groups are certain non-commutative, non-cocommutative Hopf algebras, which first appeared in the theory of quantum integrable models and later they were formalized by Drinfeld and Jimbo. In this paper we present a classification scheme for quantum groups, whose classical limit is a polynomial Lie algebra. As a consequence we obtain deformed XXX and XXZ Hamiltonians.

Keywords: Lie bialgebra, quantum group, Yang-Baxter equation, quantization, classical twist, quantum twist, integrable model.

\section{Definition of quantum groups, Lie bialgebras and twists}

In mathematics and theoretical physics, quantum groups are certain non-commutative algebras that first appeared in the theory of quantum integrable models, and which were then formalized by Drinfeld and Jimbo.

Nowadays quantum groups are one of the most popular objects in modern mathematical physics. They also play a significant role in other areas of mathematics. It turned out that quantum groups provide invariants of knots, and that they can be used in quantization of Poisson brackets on manifolds. Quantum groups gave birth to quantum geometry, quantum calculus, quantum special functions and many other "quantum" areas.

At first, quantum groups appeared to be a useful tool for the following program: Let $R$ satisfy the quantum Yang-Baxter equation (QYBE) and let $R$ be decomposable in a series in formal parameter $\hbar$. Then $r$, which is the coefficient at the first order term, satisfies the so-called classical Yang-Baxter equation (CYBE). In many cases CYBE can be solved and the problem is to extend the solutions of CYBE to solutions of QYBE.

However, the most important applications of quantum groups relate to the theory of integrable models in mathematical physics. The presence of quantum group symmetries (or so-called hidden symmetries) was the crucial point in explicit solutions of many sophisticated non-linear equations, such as Korteweg-de Vries or Sine-Gordon. Quantum groups changed and enriched representation theory and algebraic topology.

Quantum groups were defined by V. Drinfeld as Hopf algebra deformations of the universal enveloping algebras (and also their dual Hopf algebras). More exactly, we say that a Hopf algebra $\mathbf{A}$ over $\mathbf{C}[[\hbar]]$ is a quantum group (or maybe a quasi-classical quantum group) if the following conditions are satisfied:

i. The Hopf algebra $\mathbf{A} / \hbar \mathbf{A}$ is isomorphic as a Hopf algebra to the universal enveloping algebra of some Lie algebra $\mathbf{L}$.

ii. As a topological $\mathbf{C}[[\hbar]]$-module, $\mathbf{A}$ is isomorphic to $\mathbf{V}[[\hbar]]$ for some vector space $\mathbf{V}$ over $C$.

The first examples of quantum groups were quantum universal enveloping algebras $U_{q}(\mathbf{g})$, quantum affine Kac-Moody algebras $U_{q}(\hat{\mathbf{g}})$, and Yangians $Y(\mathbf{g})$.
Further, it is well-known that the Lie algebra $\mathbf{L}$ such that $\mathbf{A} / \hbar \mathbf{A}=U(\mathbf{L})$ is unique:

$$
\mathbf{L}=\{a \in \mathbf{A} / \hbar \mathbf{A}: \Delta(a)=a \otimes 1+1 \otimes a\} .
$$

If $\mathbf{A}$ is a quantum group with $\mathbf{A} / \hbar \mathbf{A}=\mathbf{U}(\mathbf{L})$, then $\mathbf{L}$ possesses a new structure, which is called a Lie bialgebra structure $\delta$, and it is given by:

$$
\delta(x)=\hbar^{-1}\left(\Delta(a)-\Delta^{o p}(a)\right) \bmod \hbar,
$$

where $a$ is an inverse image of $x$ in $\mathbf{A}$. In particular, the classical limit of $U_{q}(\mathbf{g})$ is $\mathbf{g}$, the classical limit of $U_{q}(\hat{\mathbf{g}})$ is the affine Kac-Moody algebra, which is a central extension of $\mathbf{g}\left[u, u^{-1}\right]$, and the classical limit of $Y(\mathbf{g})$ is $\mathbf{g}[u]$ (the corresponding Lie bialgebra structures will be described later).

The Lie bialgebra $(\mathbf{L}, \delta)$ is called the classical limit of $\mathbf{A}$, and $\mathbf{A}$ is a quantization of $(\mathbf{L}, \delta)$.

So, any quantum group in our sense has its classical limit, which is a Lie bialgebra, and the following natural problem arises:

Given a Lie bialgebra, is there any quantum group whose classical limit is the given Lie bialgebra? Or in other words: Can any Lie bialgebra be quantized?

In the mid 1990's P. Etingof and D. Kazhdan gave a positive answer to this problem.

Now, let $\mathbf{g}$ be a simple complex finite-dimensional Lie algebra and let $\mathbf{g}[u]$ be the corresponding polynomial Lie algebra. If you ask a physicist which quantum group is a quantization of $\mathbf{g}[u]$, you will almost certainly hear that the quantization of $\mathbf{g}[u]$ is the Yangian $Y(\mathbf{g})$.

Let us explain this in greater detail. Set $\Omega=\sum I_{\lambda} \otimes I_{\lambda}$, where $\left\{I_{\lambda}\right\}$ is an orthonormal basis of $\mathbf{g}$ with respect to the Killing form. Then it is well-known that the function

$$
\mathbf{r}(u, v)=\Gamma_{2}(u, v)=\frac{\Omega}{u-v}
$$

is a rational skew-symmetric solution of the classical Yang-Baxter equation that is

$$
\begin{aligned}
{\left[\mathbf{r}^{12}\left(u_{1}, u_{2}\right), \mathbf{r}^{13}\left(u_{1}, u_{3}\right)\right] } & +\left[\mathbf{r}^{12}\left(u_{1}, u_{2}\right), \mathbf{r}^{23}\left(u_{2}, u_{3}\right)\right] \\
+ & {\left[\mathbf{r}^{13}\left(u_{1}, u_{3}\right), \mathbf{r}^{23}\left(u_{2}, u_{3}\right)\right]=0 }
\end{aligned}
$$


More generally, we call $\mathbf{r}(u, v)$ a rational $r$-matrix if it is skew-symmetric, satisfies (2) and $\mathbf{r}(u, v)=\Gamma_{2}(u, v)+$ polynomial $(u, v)$. Sometimes $\Gamma_{2}(u, v)$ is called Yang's classical $r$-matrix. Further, the formula

$$
\delta_{2}(p(u))=\left[\Gamma_{2}(u, v), p(u) \otimes 1+1 \otimes p(v)\right]
$$

defines a Lie bialgebra structure on $\mathbf{g}[u]$, since $\Omega$ is a $\mathbf{g}$-invariant element of $\mathbf{g} \otimes \mathbf{g}$ and this implies that $\delta_{2}(p(u))$ is a polynomial in $u, v$.

The Yangian $Y(\mathbf{g})$ is precisely the quantization of this Lie bialgebra. On the other hand, it is easy to see that all rational $r$-matrices introduced in [13] define Lie bialgebra structures on $\mathbf{g}[u]$ by the formula

$$
\delta(p(u))=[\mathbf{r}(u, v), p(u) \otimes 1+1 \otimes p(v)]
$$

Of course, the next question is: Which quantum groups quantize the other "rational" Lie bialgebra structures on $\mathbf{g}[u]$ ?

Before we give an answer to this question we need the following two definitions:

Definition: Let $\delta_{1}$ be a bialgebra structure on L. Suppose $s \in \wedge^{2} \mathbf{L}$ satisfies

$$
\left[s^{12}, s^{13}\right]+\left[s^{12}, s^{23}\right]+\left[s^{13}, s^{23}\right]=\operatorname{Alt}\left(\delta_{1} \otimes i d\right)(s),
$$

where $\operatorname{Alt}(x):=x^{123}+x^{231}+x^{312}$ for any $x \in \mathbf{L}^{\otimes 3}$.

Then

$$
\delta_{2}(a):=\delta_{1}(a)+[a \otimes 1+1 \otimes a, s]
$$

defines a Lie bialgebra structure on $\mathbf{L}$.

We say that: $\delta_{2}$ is obtained from $\delta_{1}$ by twisting via $s$, and $s$ is called a classical twist.

At this point we note that any rational $r$-matrix is a twist of Yang's $r$-matrix. Let $H$ be a Hopf algebra and $F \in H \otimes H$ be an invertible element. Let $F$ satisfy

$$
F^{12}(\Delta \otimes i d) F=F^{23}(i d \otimes \Delta) F .
$$

Such $F$ is called a quantum twist. The formula

$$
\Delta_{F}(a)=F \Delta(a) F^{-1}
$$

defines a new co-multiplication on $H$.

\section{Conjecture and scheme}

Although it is clear that any quantum twist on a quantum group induces uniquely a classical twist on its classical limit, the converse statement remained unknown for a long time. It was formulated in [9] in 2004.

\section{Conjecture 1.}

Any classical twist can be extended to a quantum twist.

The conjecture was proved by G. Halbout in [3]. In particular, we can now give an answer to the question posed in the previous section: A quantum group which quantizes any rational Lie bialgebra structure on $L=\mathbf{g}[u]$ is isomorphic to the Yangian $Y(\mathbf{g})$ as an algebra and it has a twisted co-algebra structure defined by the corresponding rational solution of CYBE. However, there might exist Lie bialgebra structures on $\mathbf{g}[u]$ of a different nature, and for classification purposes one has to find all of them. Therefore, in order to classify quantum groups which have a given Lie algebra $L$ as the classical limit one has to solve the following four problems:

1. Describe all basic Lie bialgebra structures on $L$ (in other words all Lie bialgebra structures up to classical twisting).

2. Find quantum groups corresponding to the basic structures.

3. Describe all the corresponding classical twists.

4. Quantize all these classical twists.

\section{Lie bialgebra structures on the polynomial Lie algebras and their quantization}

According to the results of an unpublished paper by Montaner and Zelmanov [11], there are four basic Lie bialgebra structures on the polynomial Lie algebra $P=\mathbf{g}[u]$. Let us describe them (and, hence, we do the first step in the classification of Lie bialgebra structures on $\mathbf{g}[u])$. When it is possible we make further steps.

Case 1.

Here the one-cocycle $\delta_{1}=0$

In this case it is not difficult to show that there is a one-to-one correspondence between Lie bialgebra structures of the first type and finite-dimensional quasi-Frobenius Lie subalgebras of $\mathbf{g}[u]$. The corresponding quantum group is $U(\mathbf{g}[u])$. Classical twists can be quantized following Drinfeld's quantization of skew-symmetric constant $r$-matrices.

Case 2.

In this case the Lie bialgebra structure is described by

$$
\delta_{2}(p(u))=\left[\Gamma_{2}(u, v), p(u) \otimes 1+1 \otimes p(v)\right],
$$

where $\Gamma_{2}(u, v)$ is Yang's rational $r$-matrix.

The corresponding Lie bialgebra structures are in a one-to-one correspondence with the rational solutions of CYBE described in [13]. The corresponding quantum group is a Yangian $Y(\mathbf{g})$. Quantum twists were found for $\mathbf{g}=s l_{n}$ for $n=2,3$ in [9].

Case 3.

Here the basic Lie bialgebra structure is given by

$$
\begin{aligned}
& \delta_{3}(p(u))=\left[\Gamma_{3}(u, v), p(u) \otimes 1+1 \otimes p(v)\right] \\
& \text { with } \Gamma_{3}(u, v)=\frac{v \Omega}{v-u}+r_{D J},
\end{aligned}
$$

where $r_{D J}$ is the classical Drinfeld-Jimbo modified $r$-matrix.

This Lie bialgebra is the classical limit of the quantum group $U_{q}(g[u])$, which is a certain parabolic subalgebra of a non-twisted quantum affine algebra $U_{q}(\hat{\mathbf{g}})$ (see [15] or [10]).

There is a natural one-to-one correspondence between Lie bialgebra structures of the third type and the so-called quasi- 
-trigonometric solutions of CYBE. A complete classification of the classical twists for $\mathbf{s l}_{n}$ was presented in [9] and for the arbitrary $\mathbf{g}$ in [12]. Details on quantization of the classical twists for $\mathbf{s l}_{n}$ can be found in [9].

Case 4.

Finally, the 4-th basic Lie bialgebra structure on $\mathbf{g}[u]$ is defined as follows:

$$
\begin{aligned}
& \delta_{4}(p(u))=\left[\Gamma_{4}(u, v), p(u) \otimes 1+1 \otimes p(v)\right], \\
& \text { with } \Gamma_{4}(u, v)=\frac{\Omega}{u^{-1}-v^{-1}}=\frac{u v \Omega}{v-u} .
\end{aligned}
$$

It was proved in [14] that there is a natural one-to-one correspondence between Lie bialgebra structures of this kind and the so-called quasi-rational solutions of CYBE. The quasi-rational solutions of CYBE for $\mathbf{g}=s l_{n}$ were classified in [14]. Some ideas indicate that quasi-rational $r$-matrices do not exist for $\mathbf{g}=g_{2}, f_{4}, e_{8}$. The corresponding quantum group remains unknown, but, it is rather clear that it is related to the dual Hopf algebra of $Y(\mathbf{g})$.

\section{Some open questions}

1. Following steps 1-4 in the classification scheme, it is natural to classify quantum groups related to affine Kac-Moody algebras. A conjecture is that in this case we have only two basic types of Lie bialgebra structures and the corresponding quantum groups are quantum affine algebras and so-called doubles of $Y(\mathbf{g})$.

2. More generally: Let $L$ be a Lie algebra. Is it possible to describe the moduli space of Double $(L)$, all Lie bialgebra structures on $L$ modulo action of classical twists?

3. It is well-known that there is a 1-1 correspondence between Lie bialgebras and simply connected Poisson-Lie groups. Let us consider a Lie bialgebra $L$ and let $G$ be the corresponding Poisson-Lie group. Let $M$ be a Poisson homogeneous space that is a homogeneous space with a Poisson structure such that the multiplication

$$
m: G \times M \rightarrow M
$$

is a Poisson map.

Now, let $H$ be the quantum group which quantizes $L$.

\section{Conjecture 2.}

Let $\operatorname{Fun}(M)$ be the Poisson algebra of smooth functions on $M$. Then it can be quantized in an equivariant way, i.e., there exists an associative algebra $A$, which is a deformation of the Poisson algebra $\operatorname{Fun}(M)$, and which is a Hopf module algebra over $H$.

This conjecture is based on some results proved in [6, 7, 8]. A special case of this conjecture has been proved in [2]. More exactly, in this paper the conjecture was proved for Lie bialgebras of a special type $L=D(K)$, where $K$ is another Lie bialgebra and $L=D(K)$ is the corresponding classical double. Recent progress in related questions was achieved in [4]. Here, a result similar to the conjecture above was proved under the following assumptions: $L$ is the so-called coboundary Lie bialgebra and $L$ acts freely on $M$ (in other words $M$ is far from being a homogeneous space).

\section{Appendix: Solutions for $s l(2)$ and deformed Hamiltonians}

The aim of this section is to present concrete examples of quantum twists and the corresponding Hamiltonians following the results obtained in [5].

We consider the case $s l(2)$. Let $\sigma^{+}=e_{12}, \sigma^{-}=e_{21}$ and $\sigma^{z}=e_{11}-e_{22}$. Recall that in $s l(2)$ we have two quasi-trigonometric solutions, modulo gauge equivalence. The non-trivial solution is $X_{1}\left(z_{1}, z_{2}\right)=X_{0}\left(z_{1}, z_{2}\right)+\left(z_{1}-z_{2}\right)\left(\sigma^{+} \otimes \sigma^{+}\right)$. This solution is gauge equivalent to the following:

$$
\begin{aligned}
& X_{a, b}\left(z_{1}, z_{2}\right)=\frac{z_{2} \Omega}{z_{1}-z_{2}}+\sigma^{-} \otimes \sigma^{+}+\frac{1}{4} \sigma^{z} \otimes \sigma^{z} \\
& \quad+a\left(z_{1} \sigma^{-} \otimes \sigma^{z}-z_{2} \sigma^{z} \otimes \sigma^{-}\right)+b\left(\sigma^{-} \otimes \sigma^{z}-\sigma^{z} \otimes \sigma^{-}\right) .
\end{aligned}
$$

The above quasi-trigonometric solution was quantized in [5]. Let $\pi_{1 / 2}(z)$ be the two-dimensional vector representation of $U_{q}\left(\widehat{s l_{2}}\right)$. In this representation, the generator $e_{-\alpha}$ acts as a matrix unit $e_{21}, e_{\delta-\alpha}$ as $z e_{21}$ and $h_{\alpha}$ as $e_{11}-e_{22}$. The quantum $R$-matrix of $U_{q}\left(\widehat{s l_{2}}\right)$ in the tensor product

$$
\pi_{1 / 2}\left(z_{1}\right) \otimes \pi_{1 / 2}\left(z_{2}\right)
$$

is the following:

$$
\begin{aligned}
R_{0}\left(z_{1}, z_{2}\right)= & e_{11} \otimes e_{11}+e_{22} \otimes e_{22} \\
& +\frac{z_{1}-z_{2}}{q^{-1} z_{1}-q z_{2}}\left(e_{11} \otimes e_{22}+e_{22} \otimes e_{11}\right) \\
& +\frac{q^{-1}-q}{q^{-1} z_{1}-q z_{2}}\left(z_{2} e_{12} \otimes e_{21}+z_{1} e_{21} \otimes e_{12}\right) .
\end{aligned}
$$

Proposition 1. The $R$-matrix given by

$$
\begin{aligned}
R:= & R_{0}\left(z_{1}, z_{2}\right)+\frac{z_{1}-z_{2}}{q^{-1} z_{1}-q z_{2}}\left(\left(b+a z_{2}\right) \sigma^{z} \otimes \sigma^{-}\right. \\
& +\left(q^{-1} a z_{1}-q b\right) \sigma^{-} \otimes \sigma^{z} \\
& \left.+\left(b+a z_{2}\right)\left(q^{-1} a z_{1}+q b\right) \sigma^{-} \otimes \sigma^{-}\right)
\end{aligned}
$$

is a quantization of the quasi-trigonometric solution $X_{a, b}$.

Corollary 1. The rational degeneration

$$
\begin{aligned}
R^{F}\left(u_{1}, u_{2}\right)= & \frac{u_{1}-u_{2}}{u_{1}-u_{2}-\eta}\left(1-\eta \frac{P_{12}}{u_{1}-u_{2}}-\xi u_{2} \sigma^{z} \otimes \sigma^{-}\right. \\
& \left.+\xi\left(u_{1}-\eta\right) \sigma^{-} \otimes \sigma^{z}+\xi^{2} u_{2}\left(u_{1}-\eta\right) \sigma^{-} \otimes \sigma^{-}\right) .
\end{aligned}
$$

where $P_{12}$ denotes the permutation of factors in $C^{2} \otimes C^{2}$, is a quantization of the following rational solution of CYBE: 
$r\left(u_{1}, u_{2}\right)=\frac{\Omega}{u_{1}-u_{2}}+\xi\left(u_{1} \sigma^{-} \otimes \sigma^{z}-u_{2} \sigma^{z} \otimes \sigma^{-}\right)$.

The Hamiltonians of the periodic chains related to the twisted $R$-matrix were computed in [5]. We recall this result: We consider

$$
t(z)=\operatorname{Tr}_{0} R_{0 N}\left(z, z_{2}\right) R_{0 N-1}\left(z, z_{2}\right) \ldots R_{01}\left(z, z_{2}\right)
$$

a family of commuting transfer matrices for the corresponding homogeneous periodic chain, $\left[t\left(z^{\prime}\right), t\left(z^{\prime \prime}\right)\right]=0$, where we treat $z_{2}$ as a parameter of the theory and $z=z_{1}$ as a spectral parameter. Then the Hamiltonian

$$
H_{a, b, z_{2}}=\left.\left(q^{-1}-q\right) z \frac{\mathrm{d}}{\mathrm{d} z} t(z)\right|_{z=z_{2}} t^{-1}\left(z_{2}\right)
$$

can be computed by a standard procedure:

$H_{a, b, z_{2}}=H_{X X Z}+\sum_{k}\left(C\left(\sigma_{k}^{z} \sigma_{k+1}^{-}+\sigma_{k}^{-} \sigma_{k+1}^{z}\right)+D \sigma_{k}^{-} \sigma_{k+1}^{-}\right)$.

Here $\quad C=\frac{q-1}{2}\left(b-a z_{2} q^{-1}\right), \quad D=\left(a z_{2}+b\right)\left(q^{-1} a z_{2}+q b\right)$, $\sigma^{+}=e_{12}, \sigma^{-}=e_{21}, \sigma^{z}=e_{11}-e_{22}$ and

$H_{X X Z}=\sum_{k}\left(\sigma_{k}^{+} \sigma_{k+1}^{-}+\sigma_{k}^{-} \sigma_{k+1}^{+}+\frac{q+q^{-1}}{2} \sigma_{k}^{z} \sigma_{k+1}^{z}\right)$.

We see that, by a suitable choice of parameters $a, b$ and $z_{2}$, we can add to the XXZ Hamiltonian an arbitrary linear combination of the terms

$$
\sum_{k} \sigma_{k}^{z} \sigma_{k+1}^{-}+\sigma_{k}^{-} \sigma_{k+1}^{z} \text { and } \sum_{k} \sigma_{k}^{-} \sigma_{k+1}^{-}
$$

and the model will remain integrable.

Moreover, it was proved in [5] that the Hamiltonian

$$
H_{\eta, \xi, u_{2}}=\left.\left(\left(q^{-1}-q\right) u-q^{-1} \eta\right) \frac{\mathrm{d}}{\mathrm{d} u} t(u)\right|_{u=u_{2}} t^{-1}\left(u_{2}\right)
$$

for

$$
t(u)=\operatorname{Tr}_{0} R_{0 N}\left(u, u_{2}\right) R_{0 N-1}\left(u, u_{2}\right) \ldots R_{01}\left(u, u_{2}\right),
$$

is given by the same formula (13), where

$$
C=\xi \frac{q^{-1}-1}{2} u_{2}-\frac{q^{-1} \xi \eta}{2} \text { and } D=\xi^{2} u_{2}\left(q^{-1} u_{2}-q \eta\right) .
$$

Now it also makes sense in the XXX limit $q=1$ :

$H_{\eta, \xi, u_{2}}=H_{X X X}+\sum_{k}\left(C\left(\sigma_{k}^{z} \sigma_{k+1}^{-}+\sigma_{k}^{-} \sigma_{k+1}^{z}\right)+D \sigma_{k}^{-} \sigma_{k+1}^{-}\right)$,

where $C=-\frac{\xi \eta}{2}$ and $D=\xi^{2} u_{2}\left(u_{2}-\eta\right)$.

\section{References}

[1] Belavin, A. A., Drinfeld, V. G.: On Classical Yang-Baxter Equation for Simple Lie Algebras. Funct. An. Appl., Vol. 16 (1982), No. 3, p. 1-29.

[2] Etingof, P., Kazhdan, D.: Quantization of Poisson Algebraic Groups and Poisson Homogeneous Spaces. Symétries quantiques (Les Houches, 1995), North-Holland, Amsterdam 1998, p. 935-946.
[3] Halbout, G.: Formality Theorem for Lie Bialgebras and Quantization of Twists and Coboundary $r$-Matrices. Adv. Math. Vol. 207 (2006), p. 617-633.

[4] Halbout, G.: Quantization of $r-Z$-quasi-Poisson Manifolds and Related Modified Classical Dynamical $r$-Matrices. [arXiv math. QA/0801.2789].

[5] Khoroshkin, S., Stolin, A., Tolstoy, V.:q -Power Function over $q$-Commuting Variables and Deformed XXX and XXZ Chains. Phys. Atomic Nuclei, Vol. 64 (2001), No.12, p. 2173-2178.

[6] Karolinsky, E., Stolin, A., Tarasov, V.: Dynamical Yang-Baxter Equation and Quantization of Certain Poisson Brackets. Noncommutative Geometry and Representation Theory in Mathematical Physics. 175-182, Contemp. Math., Vol. 391 (2005), Amer. Math. Soc., Providence, RI.

[7] Karolinsky, E., Stolin, A., Tarasov, V.: Irreducible Highest Weight Modules and Equivariant Quantization. Adv. Math. Vol. 211 (2007), No. 1, p. 266-283.

[8] Karolinsky, E., Stolin, A., Tarasov, V.: Quantization of Poisson Homogeneous Spaces, Highest Weight Modules, and Kostant's Problem. In preparation.

[9] Khoroshkin, S., Pop, I., Stolin, A., Tolstoy, V.: On Some Lie Bialgebra Structures on Polynomial Algebras and Their Quantization. Preprint no. 21, 2003/2004, Mittag-Leffler Institute, Sweden.

[10] Khoroshkin, S., Pop, I., Samsonov, M., Stolin, A., Tolstoy, V.: On some Lie Bialgebra Structures on Polynomial Algebras and Their Quantization. [ArXiv math. QA/0706.1651v1]. To appear in Comm. Math. Phys.

[11] Montaner, F., Zelmanov, E.: Bialgebra Structures on Current Lie Algebras. Preprint, University of Wisconsin, Madison, 1993.

[12] Pop, I., Stolin, A.: Classification of Quasi-Trigonometric Solutions of the Classical Yang-Baxter Equation. Submitted.

[13] Stolin, A.: On Rational Solutions of Yang-Baxter Equation for $s l(n)$. Math. Scand., Vol. 69 (1991), No. 1, p. 57-80.

[14] Stolin A., Yermolova-Magnusson, J.: The 4th Structure. Czech. J. Phys., Vol. 56 (2006), No. 10/11, p. 1293-1297.

[15] Tolstoy ,V.: From Quantum Affine Kac-Moody Algebras to Drinfeldians and Yangians. Kac-Moody Lie algebras and Related topics. Contemp. Math. Vol. 343 (2004), Amer. Math. Soc., p. 349-370.

\footnotetext{
Alexander Stolin

e-mail: astolin@chalmers.se

Department of Mathematics

University of Göteborg

Göteborg, Sweden
} 